\title{
Content-Based Image Retrieval for Medical Applications with Flip-Invariant Consideration Using Low-Level Image Descriptors
}

\author{
Qusai Q. Abuein \\ Department of Computer Information Systems \\ Jordan University of Science and Technology \\ Mohammed Q. Shatnawi* \\ Department of Computer Information Systems \\ Jordan University of Science and Technology
}

\author{
Radwan Batiha \\ Department of Computer Information Systems \\ Irbid National University \\ Ahmad Al-Aiad and Suzan Amareen \\ Jordan University of Science and Technology
}

\begin{abstract}
Content-Based Image Retrieval (CBIR) has recently become one of the most attractive things in medical image research. Most existing research on CBIR focused on lowlevel image descriptors in image retrieval, which sometimes weaken the retrieval accuracy since many relevant images are not retrieved. Limited number of researches consider flipping the image on different sides. In order to fill the knowledge gap, this research focuses on considering the flipped images in retrieval based on previously implemented system that uses low-level image descriptors.

Some improvements are made on the system considering the flipped images by extracting the features of the main and flipped images. The final results showed that the proposed system outperforms the existing system. The system has proven as a powerful method in helping medical staff, physician decision makers, and students to get better results by giving wide range of needed images, and helps in reasoning and building better decisions.
\end{abstract}

Keywords-CBIR; image retrieval; feature extraction; medical images; flipped images

\section{INTRODUCTION}

The old-fashioned way of retrieving images is known as textual retrieval. This retrieval approach is basically applied by assigning specific keywords to each image in the dataset, and then retrieving the needed image or set of images according to some textual query. It is a time-consuming approach when a large number of images are searched for, and lacks accuracy due to the vocabulary mismatch since the image description is subjective.

Flipping image during the search process has been ignored by previous researchers especially in the medical field. In particular, none of the previous research in the medical field has applied image flipping in the search retrieval. Therefore, this research addresses this significant knowledge gap by enhancing the search algorithm of existing system to consider flipping images as part of the search process.

The new approach in retrieving images is Content Based Image Retrieval (CBIR). CBIR means that images can be

Identify applicable sponsor/s here. If no sponsors, delete this text box (sponsors). searched for depending on nothing but their visual content. The visual content of the image is defined as the graph, the text, the image color [1], the local and global features [2], or any other content inside the image. CBIR in other words, is mainly describing the images based on their content [3]. In CBIR, the content of the image is represented as numeric measurement [4].

CBIR can be used in searching for and retrieving images from large database collections. To retrieve these images the images must go through indexing and feature extraction methods [3]. CBIR has many methods for analyzing images; each method represents different aspects of the visual information of an image. Image searching and archival can greatly reduce the time that is consumed by using automatic image analysis tools [1].

\section{RELATED WORK}

\section{A. CBIR for Medical Applications using Low-level Image} Descriptors

A system that uses three low-level descriptors has been implemented by the authors of [5]. The system is objected to retrieve images based on their content (CBIR) for medical applications.

The system has already defined medical dataset, which contains many medical images from variant fields. The features of all images in the dataset are extracted using low-level image descriptors and the values of the features are stored in one main index file. The index of all images is consequently compared against each other. In this way, the related images will have the least distance between each other and will be retrieved together. [5]

The used features are CLD, EHD, and CEDD. The combination of these three descriptors has shown powerful results in Image retrieving, since they present color, texture, and shape, respectively.. Having such a comprehensive feature extraction system has improved the retrieval results.

\section{B. An Efficient Iconic Indexing Strategy for Image Rotation and Reflection in Image Databases}


In their effort, the authors of [4] developed a new algorithm in CBIR that considers flipped and rotated images. They not only consider the flipped and rotated images, but also the flipped and rotated objects inside the images.

The images can be classified and distinguished in two ways: the first is the low level features color, texture, and shape features, the other includes the spatial relationships between objects which is known as Retrieval by Spatial Similarity (RSS).

CBIR retrieves the images according to the content, for example, an entered query by a user can be: "show all the images that contain a circle to the right of a rectangle".

The database indices stored in the databases do not consider the flipped and rotated images, it only gives the images that satisfy the condition, where the main database may contain many images that meet the users need, these images are flipped or rotated. Therefore, an algorithm that gives all the possibilities for any index has been proposed in this research [6].

The basic idea of this research is to use symbolic projection category [8].

To extract all possible indices for an image, several transformations are made in advance. These transformations include: the original image, the image flipped horizontally, flipped vertically, rotated by 90 degrees, rotated by 180 degrees, and finally rotated by 270 degrees. Next, all these indices are stored within the dataset.

To derive all the possible indices from the original image directly, an efficient iconic indexing strategy has been presented in this research. This is done by a unique bit pattern matrix (UBP matrix). In this way, the proposed strategy will not miss the qualified images in the main dataset when the query is issued in the different orientation ways. Using rules of transformations as shown in Table I, the matrix is derived and so all the indices are generated.

TABLE I. RULES OF TRANSFORMATION: X' AND Y' ARE THE TRANSFORMED OPERATORS TO $\mathrm{X}$ AND $\mathrm{Y}$

\begin{tabular}{|l|l|l|}
\hline \multirow{2}{*}{ Functions } & \multicolumn{2}{|l|}{ Operator } \\
\cline { 2 - 3 } & x-axis & y-axis \\
\cline { 2 - 3 } & $\boldsymbol{X}$ & Y \\
\hline Rotate $90^{\circ}$ & $\mathrm{Y}$ & $\mathrm{X}^{\prime}$ \\
\hline Rotate $180^{\circ}$ & $\mathrm{X}^{\prime}$ & $\mathrm{Y}^{\prime}$ \\
\hline Rotate $270^{\circ}$ & $\mathrm{Y}^{\prime}$ & $\mathrm{X}$ \\
\hline Flip Horizontally & $\mathrm{X}^{\prime}$ & $\mathrm{Y}$ \\
\hline Flip Vertically & $\mathrm{X}$ & $\mathrm{Y}^{\prime}$ \\
\hline
\end{tabular}

Results have shown a $50 \%$ improvement compared to the traditional ways in searching. The more objects an image has, the better the results retrieved. Unfortunately, this approach is not applied on the medical images.

\section{A rotation- and Flip-invariant algorithm for} representing spatial continuity information of geographic Images in content-based Image Retrieval

This research proposes a rotation- and flip-invariant algorithm. The used images in this research are the highresolution geographic images. This algorithm is applied by representing spatial continuity information in these images. This means CBIR is applied on the geographic images. [4]

The algorithm has three main steps. The first step is to start with variogram concept: one viogaram is taken as a sample and the basic shape is captured for it. The second step is to represent the spatial continuity anisotropy for the sample shape. The final step is to extract the rotation- and flip invariant as an output. This output is the new visual property which is represented in the form of a numeric index vector.

This vector consists of a set of semi-variances at selected lags and directions. By reordering these semi-variances the original images, flipped, and rotated can be extracted. The algorithm goes through a test to confirm if the reordering can align all the image representations.

Another test for the algorithm is measuring the retrieval precision. Seven types of typical geographic entities are retrieved from an Erie County ortho-photo database, and the precision is calculated to test the effectiveness of this algorithm [4].

\section{Automatic Semantic Indexing of Medical Images}

This paper uses a grid technology to medical CBIR. The technology is used to close the gap between monolithic CBIR systems for general image retrieval purpose and the programming tools. The programming tools are used to support the development of image processing algorithms and the automatic distributed execution of them. The Grid Concept (GC) provides resource sharing. Resource sharing incorporates data sharing, access to computers, access to software. GC uses processing techniques that extract image content information into MPEG-7 format automatically, and associate them to the existing domain ontology's [7].

Several works have been done before this work, Tsechpenakisetal [ ] built a system that automatically extracts images semantics by detecting and tracking moving objects in video sequences. The domain in his work is not medical field [9].

After that, Sjo“bergetal proposed a method of content-based multimedia retrieval of objects with visual and textual properties. Objects that belong to a specific semantic class are associated with their low-level descriptors and textual features. For example, frequencies of significant keywords are extracted from audio tracks. The user provides the system with a set of sample objects that tells the system about the object he or she is looking for. The user selects the object samples from an existing database. The results showed that the retrieval performance has increased when the textual features are used. The results also showed that audio features perform very well. The domain here is not medical as well [10].

Perner [11].has developed architecture for image mining and learning semantic tagging rules. This architecture has a 
domain of specific vocabulary obtained from a domain expert or given by the domain and does not use MPEG-7 features. This architecture aims to develop the right feature extraction procedures for describing the high level semantic terms

In the developed system, the process of image segmentation, feature extraction and annotation are performed on an image by image basis. An image is selected from the same category as the ontology term and uploaded to the Visual Descriptor Extraction (VDE) tool which is developed as a plug-in to automate annotizer and presents a graphical user interface for loading and processing visual content, extraction of visual features, and association with domain ontology concepts. This technique uses pen or mouse to select the region on the image corresponding to the ontological term. Once the region of interest is selected, all image features are extracted one by one using the VDE tool. The descriptors are available as XML files in MPEG-7 standard format. The ontology term is represented as prototype instance and the links to corresponding image feature descriptors are available as another XML file in Resource Description Format (RDF) [7].

The authors [12] generate what is called Rotation and scale invariant hybrid descriptor (RSHD). The authors found that the RSHD descriptor is inherently rotation-invariant and describes the image features more efficiently. The conducted experiments of [12] show promising results.

\section{THE PROPOSED SYSTEM}

The system, which was previously introduced [5], consists of three main phases shown in Figure 1.

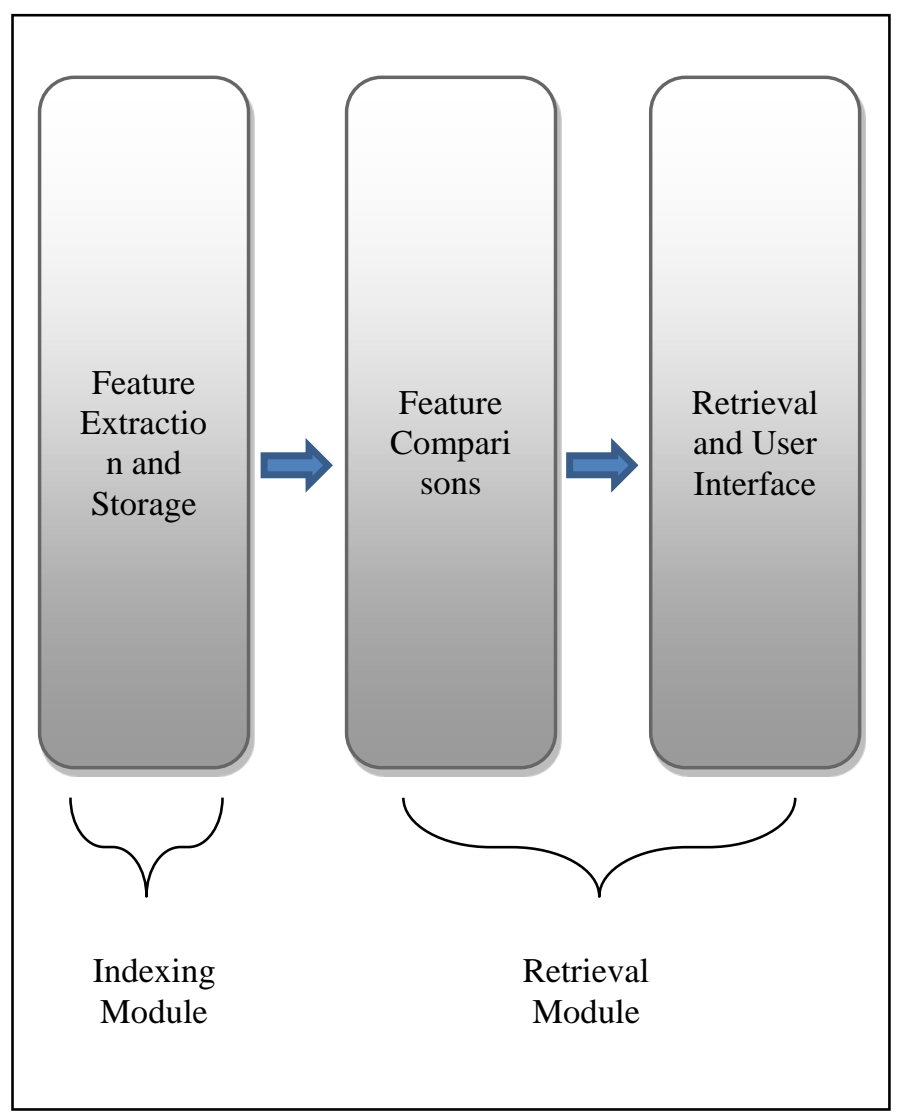

Fig. 1. Example of a figure caption. (figure caption)
The main motivation to conduct this research is the existing of the flipped images in the dataset used in the experiments introduced in [5]. One of the major limitations of the system [5] is that images that are considered as relevant are not retrieved by the introduced system in [5] because they are flipped. Figure 2 shows two relevant images each one is flipped to a different side.
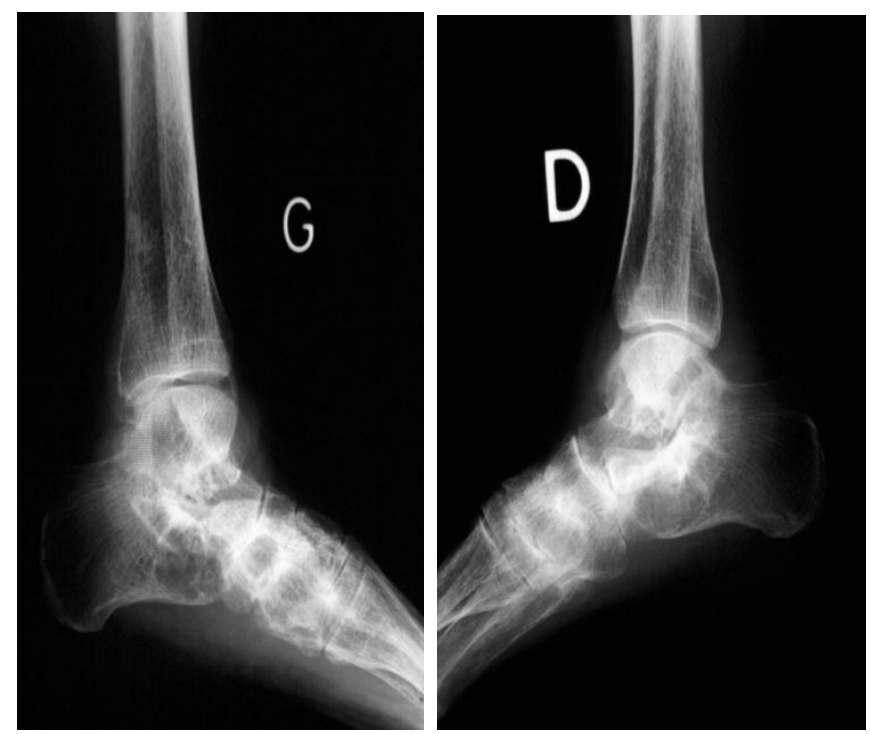

Fig. 2. Flipped Images

Figure 2 shows two X-ray images, each representing an Xray for an ankleL one is directed to the left side as shown in Figure 2 (D), and the other is directed to the right side as shown in Figure $2(\mathrm{G})$. Using the introduced system in [5], when one of the images in Figure 2 is used as an input, the other image is not retrieved in the output images. This problem can be solved by flipping each image in the dataset, so that the features of the query image is compared against the original, and the flipped image, and if any one of them matches, the image is retrieved at the retrieval phase.

The work is mainly applied in the indexing phase, when the index dataset is created. Before the three low-level features are created, the image is flipped, and then the features of the original image and the flipped image are extracted.

Figure 3 shows how image (D) of Figure 2 looks like after flipping it.

The features of the two images of Figure 3 are extracted, the index for each image is computed, and then stored in the index data file. Note that the two index values belong to the same image, the main image in the dataset remains as is. The image is flipped only in the index application. So the number of images in the main dataset remains unchanged. Only the number of rows in the index data-file is duplicated. 

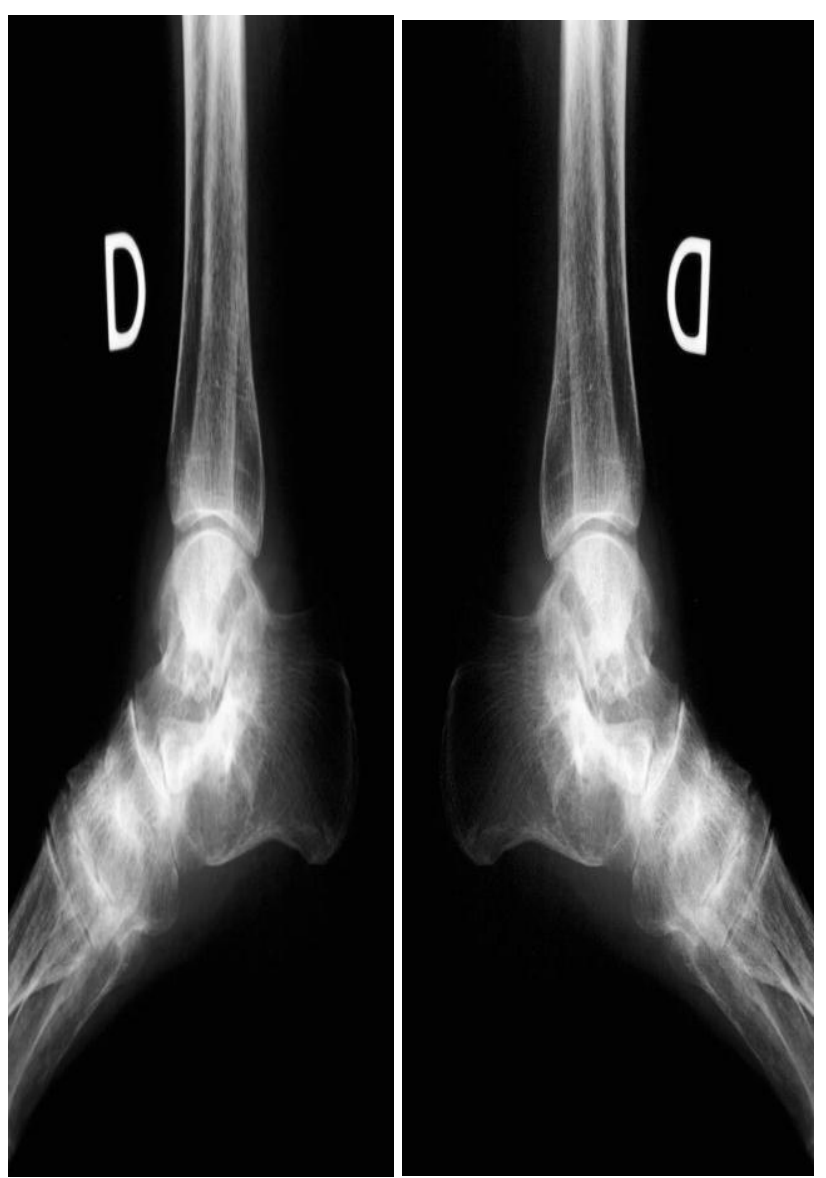

Fig. 3. Image Before and After Flipping

Now if image $(\mathrm{G})$ in Figure 2 is inserted as a query image, its features are extracted, and compared to the data-file which has the features' values of both images of Figure 3. Since Image $(\mathrm{G})$ of Figure 2 and the flipped image of Figure 3 are relevant, the feature values of them will match, and the main image in the index data-file is retrieved even if it is flipped.

Another example of flipped and related images is shown in the next figures. Figure 4 shows two images for right- and leftsection of a shoulder. The two images are relevant but are not retrieved in the same search issue due to the flipping issue. The same thing happens with Figure 5 that shows two images for a leg bent to the right and bent to the left.
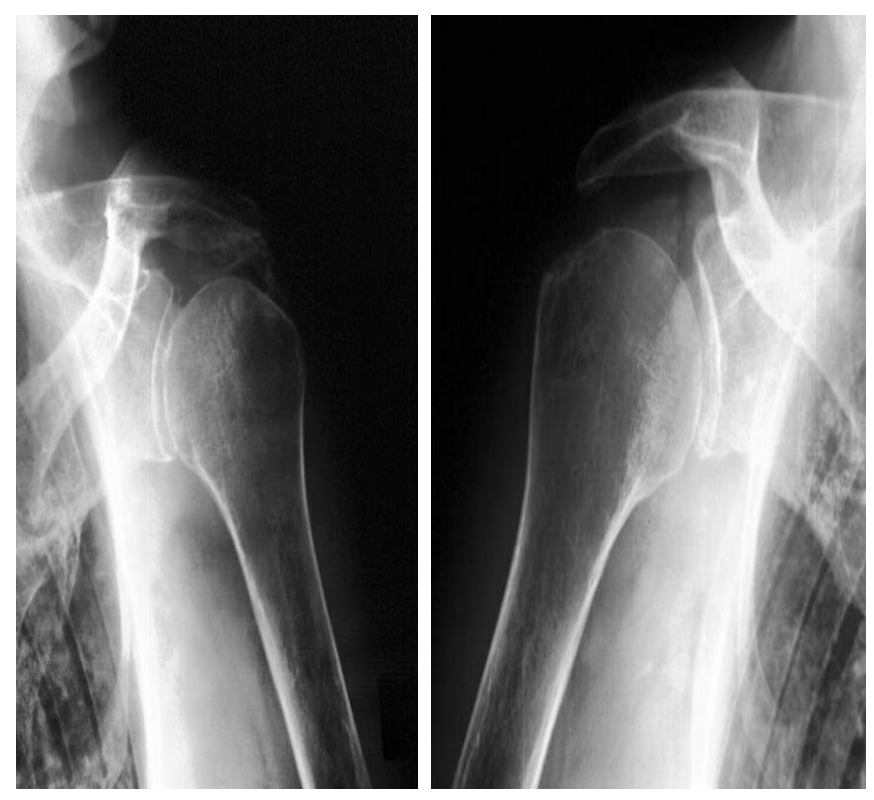

Fig. 4. Two Images for Rigth and Left Shoulder Section

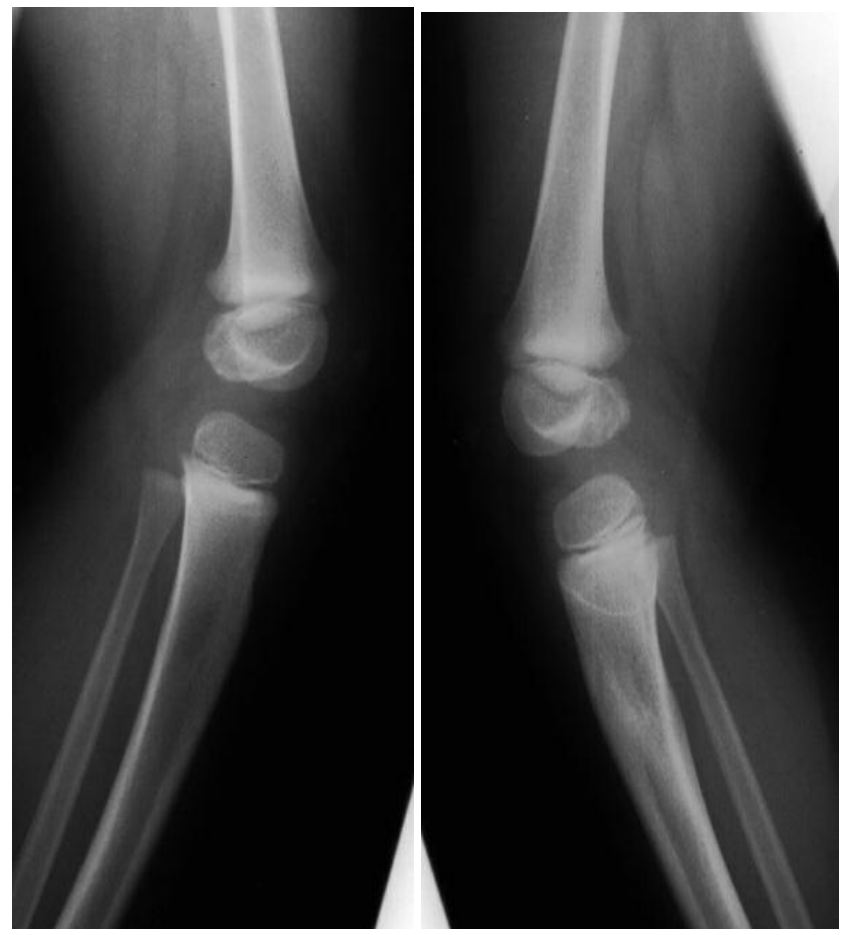

Fig. 5. Two Images for a Leg Bent to the Right and Left 


\section{EXPERIMENTS AND RESULTS}

In order to measure the performance of the proposed system, an experiment is conducted using a test of 50 cases. Then, the search is applied using the two systems. The precision and recall is compared between the two systems.

Figure 6 shows the performance results of the proposed system that considers image flipping compared to the system introduced in [5] which does not consider image flipping.

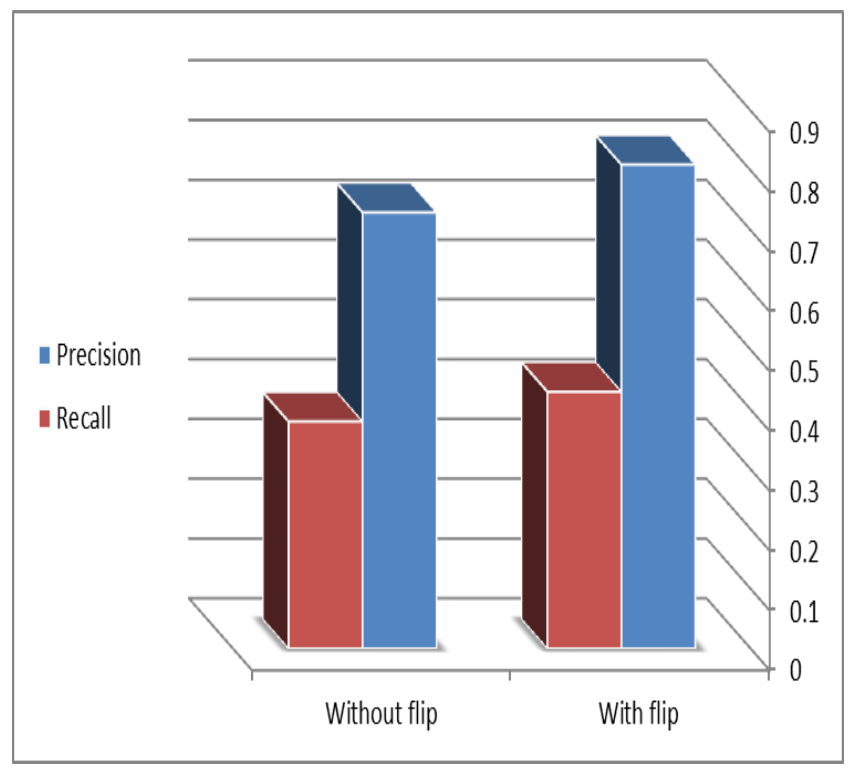

Fig. 6. Performance Comparison between the two Systems

Figure 6 shows that the improved system with flipconsideration has achieved $81 \%$ precision and $43 \%$ recall. While the previously introduced system without flippingconsideration has achieved only $73 \%$ precision and 38\% recall.

\section{CONCLUSION}

The purpose of this research is to improve an existing CBIR system by incorporating image flipping during the search retrieval process. The results show a significant improvement on system. This improvement is made by considering the flipping issue in medical images, which has been ignored by previous research.

In the different medical datasets, many flipped relevant images are found. This flipping can lead to not retrieving all the related images. A system which considers the flipped images is introduced in this research. The results have shown more accurate image retrieval system with higher precision and recall values.

Based on the analysis and the experiments that are performed in this research, it is recommend to use image flipping as a pre-processing step in the future implementation of the whole system.

\section{REFERENCES}

[1] Pavlopoulou C, Kak A, Brodley C. Content-based Image Retrieval for Medical Imagery. Proceedings SPIE Medical Imaging PACS and Integrated Medical Information Systems; 2003; San Diego CA.

[2] Müller H, Michoux N, Bandon D, Geissbuhler A. A Review of Contentbased Image Retrieval Systems in Medical Application - Clinical Benefits and Future Directions. International Journal of Medical Informatics 2004; 73: 1-23..

[3] Katarina Trojacanec, Georgina Mirceva and Danco Davcev. Application of Edge Histogram Descriptor and Region Shape Descriptor to MRIs. The Faculty of Electrical Engineering and Information Technologies, Skopje, Macedonia; 2009.

[4] ZhixiaoXie. A Rotation- and Flip-Invariant Algorithm for Representing Spatial Continuity Information of Geographic Images in Content-Based Image Retrieval. Florida Atlantic University. Computers \& Geosciences 30 (2004) 1093-1104. 2004.

[5] Suzan Amareen. Content-based Image Retrieval for Medical Applications using Low-level Image Descriptors, Master Thesis, Jordan University of Science and Technology, Defended on 31-12-2011.

[6] Wei-Horng Yeh, Ye-In Chang, An efficient iconic indexing strategy for image rotation and reflection in image databases. The Journal of Systems and Software 81 (2008) 1184-1195. National Sun Yat-Sen University, Kaohsiung, Taiwan, ROC, 2007.

[7] Gowri Allampalli-Nagaraj, Isabelle Bichindaritz. Automatic Semantic Indexing of Medical Images Using a Web Ontology Language for CaseBased Image Retrieval. Engineering Applications of Artificial Intelligence 22 p. $18-25.2009$.

[8] Petrakis. Design and evaluation of spatial similarity approaches for image retrieval. Image and Vision Computing 20 (1), 59-76. 2002.

[9] Tsechpenakis, G., Akrivas, G., Andreou, G., Stamou, G., Kollias, S. Knowledgeassisted video analysis and object detection. In: EUNITE 2002-European Symposium on Intelligent Technologies, Hybrid Systems and their Implementation on Smart Adaptive Systems. Image Video and Multimedia Laboratory, Department of Electrical and Computer Engineering, National TechnicalUniversity of Athens Retrieved October 30, 2006

[10] Sjo“ berg, M., Laaksonen, J., Po“ lla“ , M., Honkela, T. Retrieval of multimedia objects by combining semantic information from visual and textual descriptors. In: Kollias, S., Stafylopatis, A., Duch, W., Oja, E. (Eds.), Proceedings of 16th International Conference on Artificial Neural Networks. Springer, Berlin, pp. 75-83. 2006.

[11] Perner, P. Image mining: issues, framework, a generic tool and its application to medical-image diagnosis. Journal Engineering Applications of Artificial Intelligence 15 (2), 205-216. 2007.

[12] Shiv Ram Dubey, Satish Kumar Singh, Rajat Kumar Singh, "Rotation and scale invariant hybrid image descriptor and retrieval", Computers and Electrical Engineering 46 (2015) 288-302. 